\title{
Ultrasonographic index for the diagnosis of non-alcoholic steatohepatitis in patients with non-alcoholic fatty liver disease
}

\author{
Jeong Woo Kim ${ }^{1} \wedge$, Chang Hee Lee ${ }^{1} \wedge$, Baek-Hui Kim²^, Young-Sun Lee ${ }^{3} \wedge$, Soon-Young Hwang ${ }^{4}$, \\ Bit Na Park ${ }^{1}$, Yang Shin Park ${ }^{1}$
}

${ }^{1}$ Department of Radiology, Korea University Guro Hospital, Korea University College of Medicine, Seoul, Korea; ${ }^{2}$ Department of Pathology, Korea University Guro Hospital, Korea University College of Medicine, Seoul, Korea; ${ }^{3}$ Department of Internal Medicine, Korea University Guro Hospital, Korea University College of Medicine, Seoul, Korea; ${ }^{4}$ Department of Biostatistics, Korea University Guro Hospital, Korea University College of Medicine, Seoul, Korea

Contributions: (I) Conception and design: CH Lee, JW Kim; (II) Administrative support: CH Lee, JW Kim; (III) Provision of study materials or patients: BH Kim, YS Lee, CH Lee, JW Kim, BN Park, YS Park; (IV) Collection and assembly of data: BH Kim, YS Lee, JW Kim, BN Park; (V) Data analysis and interpretation: SY Hwang, JW Kim, YS Park; (VI) Manuscript writing: All authors; (VII) Final approval of manuscript: All authors.

Correspondence to: Chang Hee Lee, MD, PhD. Professor, Department of Radiology, Korea University Guro Hospital, Korea University College of Medicine, 148 Gurodong-ro, Guro-gu, Seoul 08380, Korea. Email: chlee86@korea.ac.kr.

Background: Liver biopsy is a gold standard for the diagnosis of non-alcoholic steatohepatitis (NASH), but has several disadvantages including invasiveness, high cost, and sampling error. Ultrasonography (US) is a noninvasive imaging modality widely used in non-alcoholic fatty liver disease (NAFLD) patients. This study aimed: (I) to assess the feasibility of US in the prediction of NASH and (II) to develop various US indices combining US parameters and laboratory data for the detection of NASH in NAFLD patients and to compare the diagnostic performance of them.

Methods: Sixty patients who underwent liver biopsy, gray-scale US [hepatorenal index (HRI) and shear-wave elastography (SWE)], and Fibroscan [controlled attenuation parameter (CAP) and transient elastography (TE)] for the evaluation of NASH were included. Patients were classified according to the NAFLD Activity Score (NAS) into the NASH (NAS $\geq 5$ ) and non-NASH (NAS <5) groups. The diagnostic performance of HRI, CAP, SWE, TE, and laboratory data for grading steatosis, lobular inflammation, ballooning degeneration, and fibrosis was evaluated. After the identification of laboratory data that were independently associated with NASH through univariable and multivariable logistic regression analyses, various US indices were developed by combining US parameters with or without these laboratory data. The diagnostic performance of the US indices was assessed with obtaining area under the curve (AUC) and compared using DeLong test.

Results: Twenty-five NASH and 35 non-NASH patients were included. The mean AUCs for grading steatosis were 0.871 using HRI and 0.583 using CAP. The mean AUCs for grading fibrosis and ballooning degeneration were 0.777 and 0.729 using SWE and 0.830 and 0.708 using TE, respectively. Aspartate aminotransferase (AST) was the only significant laboratory data associated with NASH (OR, 1.019; $\mathrm{P}=0.032$ ). Using AST, the mean AUCs for grading lobular inflammation and ballooning degeneration were 0.712 and 0.775 , respectively. Among various US indices, the index consisting of gray-scale US parameters (SWE and HRI) and AST showed the best diagnostic performance for the detection of NASH in NAFLD patients (AUC $=0.806$ ).

Conclusions: The index combining gray-scale US parameters and AST is useful for the detection of

^ ORCID: Jeong Woo Kim, 0000-0003-1580-1006; Chang Hee Lee, 0000-0003-3381-2227; Baek-Hui Kim, 0000-0001-6793-1991; YoungSun Lee, 0000-0001-6396-0859; Soon-Young Hwang, 0000-0001-7474-1803. 
NASH and may be used to exclude the need for liver biopsy in NAFLD patients.

Keywords: Non-alcoholic fatty liver disease (NAFLD); non-alcoholic steatohepatitis (NASH); ultrasonography; index

Submitted Sep 08, 2021. Accepted for publication Dec 06, 2021; Published online: 01 Jan 2022.

doi: $10.21037 /$ qims-21-895

View this article at: https://dx.doi.org/10.21037/qims-21-895

\section{Introduction}

Non-alcoholic fatty liver disease (NAFLD) is a leading cause of chronic liver disease worldwide, with the global prevalence of $24 \%(1,2)$. The prevalence is gradually increasing not only in the Western countries but also in Asian countries $(2,3)$. Non-alcoholic steatohepatitis (NASH) is a part of the NAFLD spectrum, which histologically features lobular inflammation and hepatocyte ballooning degeneration with or without fibrosis. Progression to the advanced fibrosis, and ultimately cirrhosis, was reported in about $25 \%$ of $\mathrm{NASH}$ patients $(4,5)$. In addition, NASH can increase the risk of esophageal varices, hepatocellular carcinoma (HCC), and cardiovascular mortality (4-7). In the early stage of NAFLD without significant fibrosis, lifestyle modification and pharmacotherapy can improve inflammation and steatosis $(8,9)$. Therefore, it is clinically important to distinguish NASH from NAFLD because earlier diagnosis and more aggressive treatment of NASH can reduce overall mortality $(10,11)$. Liver biopsy, the current gold standard for diagnosing NASH, has drawbacks including the risk of bleeding and high cost. In addition, since the histologic components of NASH are not distributed evenly in the liver, sampling error is the biggest limitation of liver biopsy (5).

There have been several studies that used non-invasive methods to distinguish NASH from NAFLD. A non-invasive imaging technique known as elastography can detect hepatic fibrosis and cirrhosis in NAFLD patients (12). Elastography measures liver stiffness through the analysis of mechanical waves transmitted into the hepatic tissue and is implemented on magnetic resonance (MR) or ultrasound (US) system. USbased elastography has the advantages of having a shorter acquisition time, being more convenient, associated with lower cost, and more available than MR-based elastography. Therefore, US-based elastography, including transient elastography (TE), two-dimensional shear-wave elastography (2D-SWE), and point shear-wave elastography (p-SWE, acoustic radiation force impulse) are more widely used in clinical setting $(13,14)$. TE is a one-dimensional technique performed using the Fibroscan device (EchoSens, Paris, France) (15). 2D-SWE is a newer US-based elastography technique, which can be performed using a conventional US scanner and enables real-time imaging (16).

There are several imaging techniques that can be used to assess another major component of NASH, the hepatic fat. Among them, gray-scale US can detect moderate to severe hepatic steatosis with a fairly good diagnostic accuracy, but demonstrates a limited performance in detecting mild hepatic steatosis with a reported sensitivity of $61-65 \%$ $(17,18)$. To overcome this limitation, several US-based quantitative assessments, including the hepatorenal index (HRI) and the controlled attenuation parameter (CAP) have been developed. The HRI is defined as the ratio of echo intensity of the liver parenchyma to that of the renal cortex. It can be obtained during conventional gray-scale US examinations. The CAP measured by the Fibroscan device can be obtained simultaneously with the TE (19).

Considering the invasiveness and sampling error of liver biopsy, the need for this procedure may be reduced if patients who do not need liver biopsy can be excluded during routine clinical US. We planned to develop US indices that can predict NASH in NAFLD patients, combining US parameters with laboratory data. The purpose of our study was: (I) to assess the feasibility of US in predicting NASH in NAFLD patients and (II) to develop various US indices using gray-scale US parameters (SWE and HRI), Fibroscan parameters (TE and CAP), and laboratory data for the detection of NASH in NAFLD patients and to compare the diagnostic performance of them.

We present the following article in accordance with the STARD reporting checklist (available at https://dx.doi. org/10.21037/qims-21-895).

\section{Methods}

\section{Patients}

The study was conducted in accordance with the 


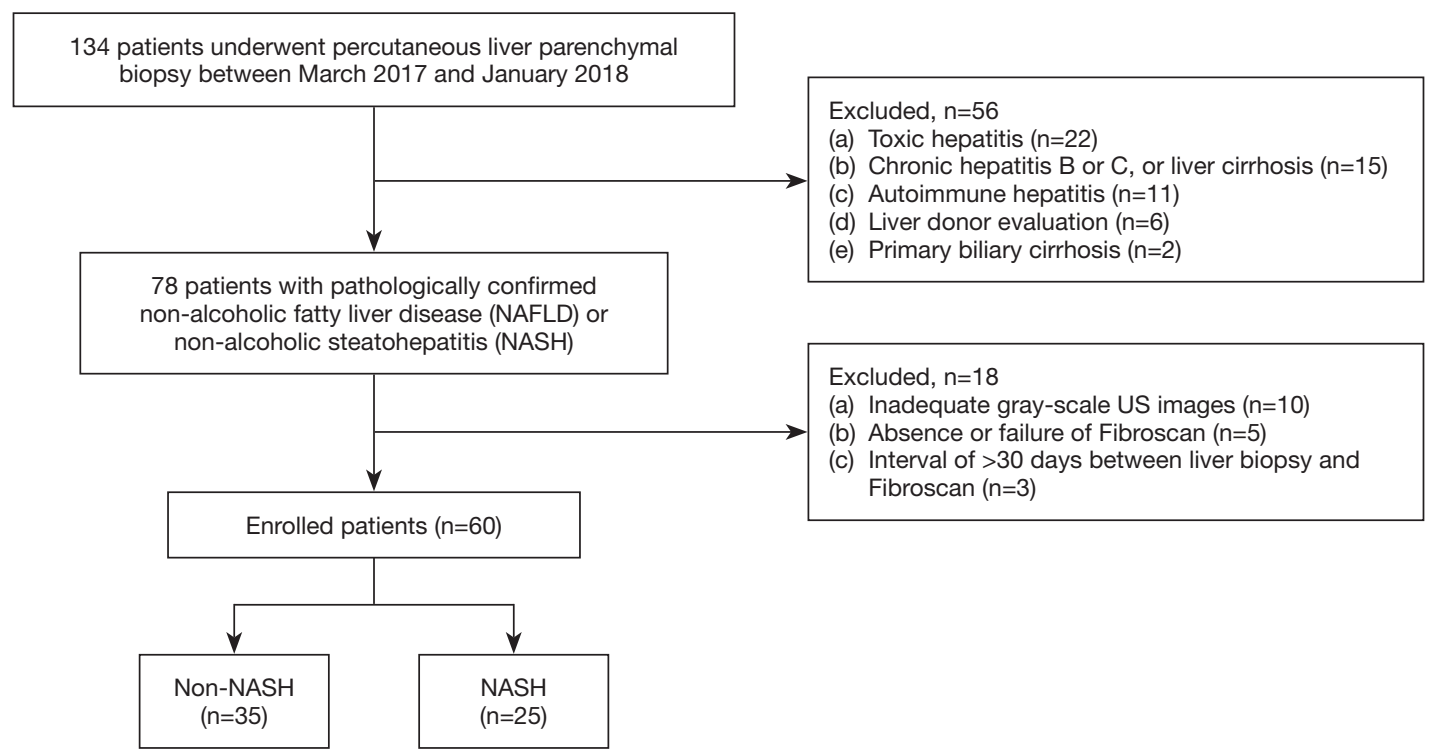

Figure 1 Flow chart of the study population.

Declaration of Helsinki (as revised in 2013). This was a retrospective study following Health Insurance Portability and Accountability Act (HIPAA). It was approved by our institutional review board (IRB No. KUGH16184), which allowed to waive the requirement for informed consent. A hundred and thirty-four patients who underwent percutaneous liver biopsy were consecutively recruited from March 2017 to January 2018. Among these patients, 56 patients were excluded due to diagnosis other than NAFLD or NASH: (I) toxic hepatitis $(\mathrm{n}=22)$; (II) chronic hepatitis B or $\mathrm{C}$, or liver cirrhosis ( $\mathrm{n}=15$ ); (III) autoimmune hepatitis ( $\mathrm{n}=11$ ); (IV) liver donor evaluation $(\mathrm{n}=6)$; and $(\mathrm{V})$ primary biliary cirrhosis $(\mathrm{n}=2)$. In addition, 18 patients were excluded due to (I) inadequate gray-scale US images $(\mathrm{n}=10)$; (II) absence or failure of Fibroscan ( $\mathrm{n}=5$ ); and (III) interval of more than 30 days between liver biopsy and Fibroscan $(\mathrm{n}=3)$. Finally, 60 patients who underwent liver biopsy, grayscale US, and Fibroscan for the evaluation of NASH were included (28 men and 32 women; mean age, $50.9 \pm 13.4$ years) (Figure 1).

\section{Histopathological evaluation}

Percutaneous liver biopsy was performed at the right hepatic lobe using an 18-gauge semi-automatic needle (TSK Laboratory, Tochigi, Japan). At least two cores of hepatic tissue were obtained, and the biopsy specimens were then reviewed by a pathologist (B.K., more than 15 years of experience) who was blinded to the patients' radiologic and clinical data. Biopsy specimens were classified according to the NAFLD Activity Score (NAS) system into the NASH (NAS score $\geq 5$ ) or the non-NASH category (20). The NAS is a semi-quantitative score, which is the unweighted sum of steatosis (0-3), hepatocyte ballooning degeneration (0-2), and lobular inflammation (0-3) (Table 1).

\section{Shear-wave elastography with bepatorenal index (Gray- scale US)}

All patients underwent gray-scale US examinations using a US machine (Aplio 500; Toshiba Medical System, Otawara, Japan) with a $5-\mathrm{MHz}$ convex transducer just before the percutaneous liver biopsy. The US examinations were performed by one of five abdominal radiologists with at least 1 year of experience in performing real-time elastography. SWE was performed in the right hepatic lobe through the intercostal spaces. A $2 \times 2-\mathrm{cm}$ SWE box was placed in the liver parenchyma, while avoiding large vessels, and the upper margin of the SWE box was placed 1.5-2.0 cm away from the liver surface (Glisson capsule). Liver stiffness (LS) measurements were obtained with a region-of-interest (ROI) of $10 \mathrm{~mm}^{2}$ in the SWE box at the area displaying the most homogeneous stiffness and neither movement nor pressure artifacts. We measured at least 10 ROIs and used the median LS values (in kilopascals) as representative values.

To calculate the HRI, two radiologists (JWK, and CHL), 
Table 1 Nonalcoholic fatty liver disease (NAFLD) Activity Score (NAS)

\begin{tabular}{|c|c|}
\hline Grade & Description \\
\hline \multicolumn{2}{|c|}{$\begin{array}{l}\text { Steatosis: amount of large or medium-sized lipid droplets without } \\
\text { foamy microvesicles }\end{array}$} \\
\hline so & $<5 \%$ \\
\hline S1 & $5-33 \%$ \\
\hline $\mathrm{S} 2$ & $33-66 \%$ \\
\hline S3 & $>67 \%$ \\
\hline \multicolumn{2}{|c|}{ Hepatocyte ballooning degeneration } \\
\hline B0 & None \\
\hline B1 & Few balloon cells \\
\hline B2 & Many cells \\
\hline \multicolumn{2}{|c|}{ Lobular inflammation } \\
\hline LO & None \\
\hline L1 & $<2$ foci/200 fields \\
\hline L2 & $2-4$ foci/200 fields \\
\hline L3 & $\geq 4$ foci/200 fields \\
\hline \multicolumn{2}{|c|}{ Fibrosis } \\
\hline F0 & No fibrosis \\
\hline \multicolumn{2}{|l|}{$\mathrm{F} 1$} \\
\hline $\mathrm{F} 1 \mathrm{a}$ & Zone 3 mild perisinusoidal fibrosis \\
\hline $\mathrm{F} 1 \mathrm{~b}$ & Zone 3 moderate perisinusoidal fibrosis. \\
\hline F1c & Portal/Periportal fibrosis only \\
\hline $\mathrm{F} 2$ & Zone 3 perisinusoidal and portal/periportal fibrosis \\
\hline F3 & Bridging fibrosis \\
\hline $\mathrm{F} 4$ & Cirrhosis \\
\hline
\end{tabular}

NAS = Steatosis $(S)+$ Ballooning degeneration $(B)+$ Lobular inflammation (L).

who were blinded to the clinical data and pathology results, performed quantitative measurement on still images at the PACS workstation using a method described by Shiralkar et al. (21). Circular ROIs $\left(0.5-1.5 \mathrm{~cm}^{2}\right)$ were manually drawn at the liver parenchyma and renal cortex on the same image, placing them at the same depth and avoiding large ducts, vessels, renal collecting systems, and focal lesions. The HRI was calculated by dividing the liver ROI value by the renal cortex ROI value. This measurement was repeated three times on three different images and the mean value was recorded.

\section{Transient elastography with controlled attenuation parameter (Fibroscan)}

All patients underwent TE examinations using the Fibroscan (Echosens, Paris, France) equipped with $M$ and $\mathrm{XL}$ probes by an experienced operator, who was blinded to the clinical information. The transducer was positioned on the skin of the intercostal spaces over the right hepatic lobe. Measurements were repeated up to 20 times until a minimum of 10 valid LS values in kilopascals $(\mathrm{kPa})$ were obtained. To evaluate the variability, the ratio (IQR/M) of the interquartile range (IQR) of LS value to the median value $(M)$ was calculated. Technical failure was defined as no LS measurement or unreliable measurement (success rate $<60 \%$ and/or IQR/M $>30 \%$ ). At the same time, the hepatic steatosis was assessed using the CAP value in $\mathrm{dB} / \mathrm{m}$.

\section{Statistical analysis}

For laboratory and clinical data, the chi-square $\left(\chi^{2}\right)$ or Fisher's exact test was used to compare categorical variables, and the independent-sample $t$-test or Mann-Whitney $\mathrm{U}$ test was used to compare continuous variables between the NASH and non-NASH groups. The chi-square $\left(\chi^{2}\right)$ or Fisher's exact test was also used to compare the distribution of each histopathologic component.

Interobserver agreement of two radiologists in calculating HRI was assessed using $\kappa$-statistics with $\kappa$-value graded as excellent $(0.81-1.0)$, good $(0.61-0.80)$, moderate (0.41-0.60), fair (0.21-0.40), and poor (0-0.20).

To evaluate the diagnostic performances of HRI, CAP, SWE, TE, and laboratory data for grading each histopathological component (steatosis, fibrosis, lobular inflammation, and ballooning degeneration), ROC curve analyses were performed and the areas under the curve (AUCs) were obtained with their 95\% confidence intervals (CIs). DeLong tests were used to compare AUCs for grading steatosis between HRI and CAP, and to compare AUCs for grading fibrosis between SWE and TE.

The US index was developed in two steps. First, significant laboratory data for predicting NASH was selected by univariable and multivariable logistic regression analyses (22). Factors in univariable logistic regression analysis with a $\mathrm{P}$ value $<0.05$ were further analyzed by using a multivariable logistic regression analysis (22). Second, various US indices were developed through multivariable logistic regression analysis, combining US parameters with laboratory data or combining only US parameters 


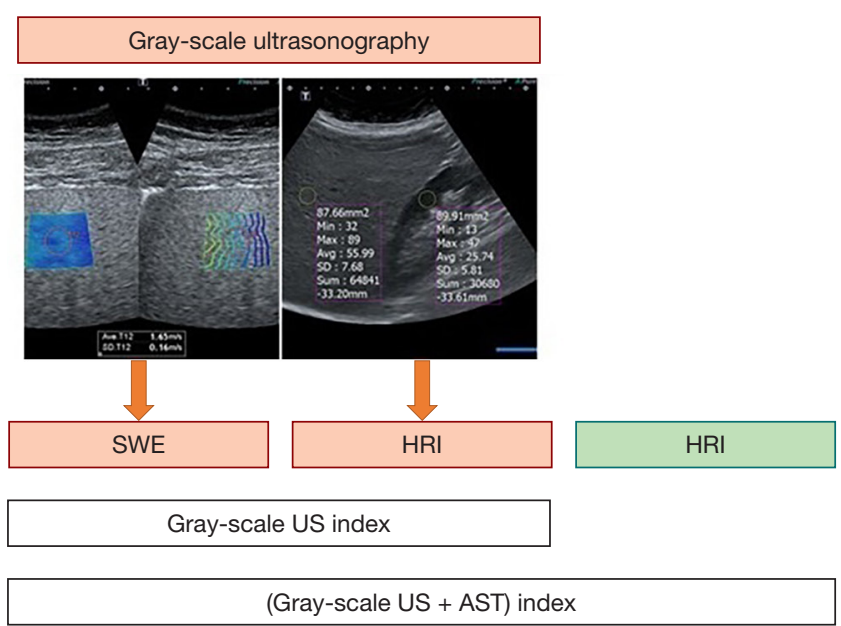

(Fibroscan + AST) index

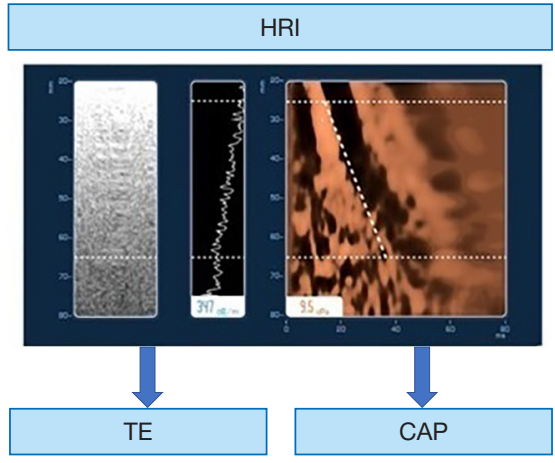

Fibroscan index

(Gray-scale US + Fibroscan) index

(Gray-scale US + Fibroscan + AST) index

Figure 2 Development of non-invasive ultrasonography (US) indices. A variety of US indices were developed, combining US parameters [Gray-scale US parameters (SWE and HRI) and/or Fibroscan parameters (TE and CAP)] with or without AST, which was the only significant factor associated with NASH determined by univariable and multivariable logistic regression analyses. SWE, shear-wave elastography; HRI, hepatorenal index; TE, transient elastography; CAP, controlled attenuation parameter; AST, aspartate aminotransferase; NASH, non-alcoholic steatohepatitis.

(Figure 2) (22). ROC curve analyses were performed to assess the diagnostic performances of the US indices and the AUCs were obtained with their 95\% CIs. The optimal cutoff values corresponding to the maximal sum of the sensitivity and specificity were calculated. The DeLong test was used to compare AUCs of the US indices.

A $P$ value $<0.05$ was considered statistically significant. All statistical analyses were performed using commercially available software programs, SPSS version 25 (IBM Corp., Armonk, NY, USA) and MedCalc version 18.6 (MedCalc Software, Ostend, Belgium).

\section{Results}

\section{Patients}

There was no adverse event during and after liver biopsy in all patients. Patients were classified into the NASH $(n=25 ; 8$ men and 17 women; mean age, $53.0 \pm 13.6$ years) and non-NASH $(\mathrm{n}=35 ; 20$ men and 15 women; mean age, $49.3 \pm 13.2$ years) groups based on the NAS system. The distribution of histopathologic components is summarized in Table 2.
The grades of each component tended to be higher in the NASH group than in the non-NASH group. The serum aspartate aminotransferase (AST) and alkaline phosphatase (ALP) levels were significantly higher, and the serum triglycerides (TG) was significantly lower in the NASH group than in the non-NASH group $(\mathrm{P}=0.003,0.028$, and 0.013 , respectively). Other clinical and laboratory data did not show any significant difference between the two groups (Table 2).

\section{US parameters and histopathologic components}

The mean values $(\mathrm{kPa})$ of SWE in the F0, F1, F2, F3, and F4 groups were $10.5 \pm 2.9,9.4 \pm 1.6,10.7 \pm 3.0,15.2 \pm 6.9$, and $18.4 \pm 6.8$, respectively. The mean values $(\mathrm{kPa})$ of TE in the F0, F1, F2, F3, and F4 groups were 9.0 $03.1,9.6 \pm 2.2$, $12.3 \pm 5.3,21.3 \pm 14.3$, and $26.4 \pm 16.9$, respectively. The diagnostic performances of SWE and TE for grading fibrosis are summarized in Table 3. Using SWE, the AUCs for grading $\geq \mathrm{F} 1, \geq \mathrm{F} 2, \geq \mathrm{F} 3$, and $\geq \mathrm{F} 4$ were $0.653,0.747$, 0.861 , and 0.846 , respectively. Using TE, the AUCs were $0.733,0.828,0.869$, and 0.891 , respectively. The 
Table 2 Baseline characteristics

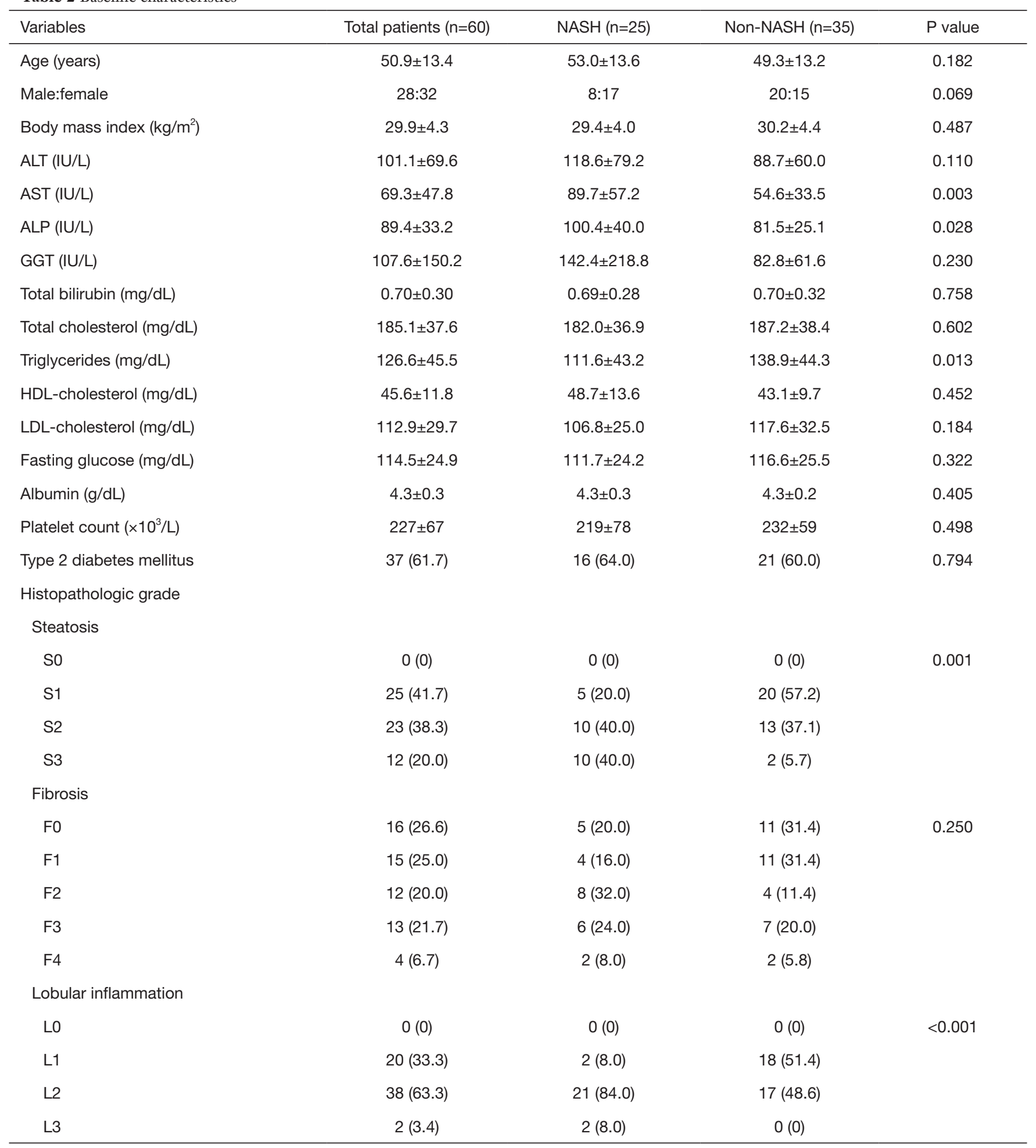

Table 2 (continued) 
Table 2 (continued)

\begin{tabular}{|c|c|c|c|c|}
\hline Variables & Total patients $(n=60)$ & NASH $(n=25)$ & Non-NASH $(n=35)$ & $P$ value \\
\hline BO & $34(56.7)$ & $6(24.0)$ & $28(80.0)$ & $<0.001$ \\
\hline B1 & $9(15.0)$ & $4(16.0)$ & 5 (14.3) & \\
\hline B2 & $17(28.3)$ & $15(60.0)$ & $2(5.7)$ & \\
\hline
\end{tabular}

Continuous variables are presented as mean \pm standard deviation and were compared using the independent sample $t$-test or Mann-Whitney $U$-test. Categorical variables are presented as numbers (\%) and were compared using the chi-square $\left(\chi^{2}\right)$ test. ALT, alanine aminotransferase; AST, aspartate aminotransferase; ALP, alkaline phosphatase; GGT, $\gamma$-glutamyltransferase; HDL, high density lipoprotein; LDL, low density lipoprotein.

diagnostic performances for grading hepatic fibrosis were not significantly different between SWE and TE $(\mathrm{p}=$ $0.321,0.302,0.963$, and 0.668 for $\geq F 1, \geq F 2, \geq F 3$, and $\geq F 4$, respectively).

SWE and TE also showed high diagnostic performance for grading ballooning degeneration (mean AUC, 0.729 and 0.708 , respectively), but relatively low diagnostic performance for grading lobular inflammation (mean AUC, 0.625 and 0.522 , respectively) (Table 3).

Interobserver agreement for calculating HRI was excellent ( $\kappa=0.949 ; 95 \%$ CI: $0.914-0.970, \mathrm{P}<0.001)$. The mean values of the HRI in the $\mathrm{S} 1, \mathrm{~S} 2$, and $\mathrm{S} 3$ groups were $1.6 \pm 0.4,2.2 \pm 0.4$, and $3.2 \pm 2.0$, respectively. The mean values $(\mathrm{dB} / \mathrm{m})$ of $\mathrm{CAP}$ in the $\mathrm{S} 1, \mathrm{~S} 2$, and $\mathrm{S} 3$ groups were $308.2 \pm 51.9,323.5 \pm 48.6$, and $325.5 \pm 29.0$, respectively. The diagnostic performances of the CAP and HRI for grading steatosis are summarized in Table 3. HRI showed higher diagnostic performance than CAP for grading both $\geq \mathrm{S} 2$ and $\geq \mathrm{S} 3$ steatosis (AUC for $\geq \mathrm{S} 2,0.890$ vs. $0.616, \mathrm{P}=0.001$ and $\mathrm{AUC}$ for $\geq \mathrm{S} 3,0.851$ vs. $0.549, \mathrm{P}=0.001)$.

\section{Laboratory data and histopathologic components}

Of the laboratory data, AST was identified as the only significant factor associated with NASH by univariable and multivariable logistic regression analyses. The mean values (IU/L) of serum AST level in the L1, L2, and L3 groups were $56.5 \pm 43.8,74.2 \pm 49.0$, and $103.0 \pm 55.2$, respectively. The diagnostic performance of AST for grading lobular inflammation are summarized in Table 3. Using AST, the AUCs for grading $\geq \mathrm{L} 2$ and $\geq \mathrm{L} 3$ were 0.664 and 0.759 , respectively.

The mean values (IU/L) of serum AST level in the B0, B1, and B2 groups were $50.1 \pm 22.0,80.4 \pm 54.5$, and $101.7 \pm 62.9$, respectively. The diagnostic performance of
AST for grading ballooning degeneration are summarized in Table 3. Using AST, the AUCs for grading $\geq \mathrm{L} 1$ and $\geq \mathrm{L} 2$ were 0.769 and 0.780 , respectively.

AST demonstrated relatively low diagnostic performance for grading fibrosis (mean AUC, 0.606) (Table 3).

\section{US index for the diagnosis of $\mathrm{NASH}$}

The results of univariable logistic regression analysis of laboratory data are shown in Table 4. AST [odds ratio (OR), 1.021; $\mathrm{P}=0.015]$, ALP (OR, 1.019; $\mathrm{P}=0.037)$, and TG (OR, $0.988 ; \mathrm{P}=0.030$ ) were the significant factors associated with NASH. These factors were entered into the multivariable logistic regression analysis, revealing that AST was the only independent factor for predicting NASH (OR, 1.019; $\mathrm{P}=0.032$ ) (Table 4). Therefore, various US indices were developed by combining US parameters with or without AST. Table 5 shows the diagnostic performance of various US indices. Among the US indices, the index consisting of gray-scale US parameters (SWE and HRI) and AST demonstrated the best diagnostic performance. The index was obtained as follows based on the multivariable logistic regression model using SWE, HRI, and AST (Figure 3).

$$
\log (P / 1-P)=0.257 \times S W E+1.246 \times H R I+0.017 \times A S T-7.039
$$

This can be expressed in the form of predicted probability with a value between 0 and 1 .

$$
P=\frac{e^{0.257 \times S W E+1.246 \times H R I+0.017 \times A S T-7.039}}{1+e^{0.257 \times S W E+1.246 \times H R I+0.017 \times A S T-7.039}}
$$

ROC curve analysis of this index revealed an AUC of 0.806 with an optimal cutoff value of 0.4213 . The corresponding sensitivity and specificity were 68.0 and $80.0 \%$, respectively (Table 5, Figure 4). The US index 
Table 3 Diagnostic performance of shear-wave elastography (SWE), transient elastography (TE), hepatorenal index (HRI), controlled attenuation parameter (CAP), and aspartate aminotransferase (AST) for grading each histopathologic component

\begin{tabular}{|c|c|c|c|c|c|c|c|}
\hline Variables & Components & Grade & Mean AUC & AUC & Cut-off value $^{\dagger}$ & Sensitivity (\%) & Specificity (\%) \\
\hline \multirow{6}{*}{ SWE } & \multirow{3}{*}{ Fibrosis } & $\geq \mathrm{F} 2$ & & $0.747(0.611,0.854)$ & 11.9 & $67.9(47.7,84.1)$ & $77.8(57.7,91.3)$ \\
\hline & & $\geq \mathrm{F} 3$ & & $0.861(0.742,0.940)$ & 12.7 & $76.5(50.1,93.0)$ & $81.6(65.7,92.2)$ \\
\hline & & $\geq \mathrm{F} 4$ & & $0.846(0.730,0.926)$ & 14.9 & $75.0(19.4,99.4)$ & $92.9(82.7,98.0)$ \\
\hline & $\begin{array}{l}\text { Lobular } \\
\text { inflammation }\end{array}$ & $\geq \mathrm{L} 3$ & & $0.746(0.617,0.849)$ & 12.9 & $100.0(15.8,100.0)$ & 72.4 (59.1, 83.3) \\
\hline & \multirow{2}{*}{$\begin{array}{c}\text { Ballooning } \\
\text { degeneration }\end{array}$} & $\geq \mathrm{B} 1$ & 0.729 & $0.640(0.505,0.760)$ & 14.6 & $30.8(14.3,51.8)$ & $97.1(84.7,99.9)$ \\
\hline & & $\geq \mathrm{B} 2$ & & $0.817(0.696,0.905)$ & 9.9 & $94.1(71.3,99.9)$ & $60.5(44.4,75.0)$ \\
\hline \multirow[t]{6}{*}{ TE } & \multirow[t]{2}{*}{ Fibrosis } & $\geq \mathrm{F} 1$ & 0.830 & $0.733(0.603,0.839)$ & 9.6 & $77.3(62.2,88.5)$ & $62.5(35.4,84.8)$ \\
\hline & & $\geq \mathrm{F} 4$ & & $0.891(0.783,0.957)$ & 13.0 & $100.0(39.8,100.0)$ & $78.6(65.6,88.4)$ \\
\hline & \multirow{2}{*}{$\begin{array}{l}\text { Lobular } \\
\text { inflammation }\end{array}$} & $\geq \mathrm{L} 2$ & 0.522 & $0.531(0.397,0.661)$ & 10.0 & $70.0(53.5,83.4)$ & $50.0(27.2,72.8)$ \\
\hline & & $\geq$ L3 & & $0.513(0.380,0.644)$ & 7.5 & $50.0(1.3,98.7)$ & $87.9(76.7,95.0)$ \\
\hline & \multirow{2}{*}{$\begin{array}{l}\text { Ballooning } \\
\text { degeneration }\end{array}$} & $\geq \mathrm{B} 1$ & 0.708 & $0.657(0.523,0.774)$ & 11.7 & $53.9(33.4,73.4)$ & $79.4(62.1,91.3)$ \\
\hline & & $\geq \mathrm{B} 2$ & & $0.758(0.630,0.859)$ & 11.8 & $64.7(38.3,85.8)$ & $81.4(66.6,91.6)$ \\
\hline \multirow[t]{2}{*}{ HRI } & \multirow[t]{2}{*}{ Steatosis } & $\geq \mathrm{S} 2$ & 0.871 & $0.890(0.783,0.956)$ & 1.80 & $88.6(73.3,96.8)$ & $88.0(68.8,97.5)$ \\
\hline & & $\geq \mathrm{S} 3$ & & $0.851(0.735,0.930)$ & 2.31 & $83.3(51.6,97.9)$ & $81.3(67.4,91.1)$ \\
\hline CAP & Steatosis & $\geq \mathrm{S} 2$ & 0.583 & $0.616(0.481,0.739)$ & 291 & $80.0(63.1,91.6)$ & $52.0(31.3,72.2)$ \\
\hline \multirow{4}{*}{ AST } & \multirow[t]{4}{*}{ Fibrosis } & $\geq \mathrm{F} 1$ & 0.606 & $0.686(0.553,0.800)$ & 64 & $52.3(36.7,67.5)$ & $87.5(61.7,98.4)$ \\
\hline & & $\geq \mathrm{F} 2$ & & $0.613(0.478,0.736)$ & 74 & $44.8(26.4,64.3)$ & $80.7(62.5,92.5)$ \\
\hline & & $\geq \mathrm{F} 3$ & & $0.593(0.458,0.718)$ & 75 & $52.9(27.8,77.0)$ & $79.1(64.0,90.0)$ \\
\hline & & $\geq \mathrm{F} 4$ & & $0.533(0.400,0.663)$ & 16 & $25.0(0.6,80.6)$ & $100.0(93.6,100.0)$ \\
\hline
\end{tabular}

Data in brackets are $95 \%$ confidence intervals. ${ }^{\dagger}$, units of SWE, TE, and CAP are kilopascal $(\mathrm{kPa})$, kilopascal $(\mathrm{kPa})$, and decibles/meter $(\mathrm{dB} / \mathrm{m})$, respectively. AUC, area under the curve.

consisting of gray-scale US parameters and AST showed higher AUC than other indices with a significant difference compared to the Fibroscan index (AUC, 0.806 vs. 0.687, $\mathrm{P}=0.04$ ) and no statistically significant difference from other indices.

\section{Discussion}

Our study demonstrated that various US indices combining gray-scale US parameters (SWE and HRI), Fibroscan parameters (TE and CAP), and AST showed good diagnostic performance for the detection of NASH in 
Table 4 Univariable and multivariable logistic regression analyses of laboratory data associated with non-alcoholic steatohepatitis (NASH)

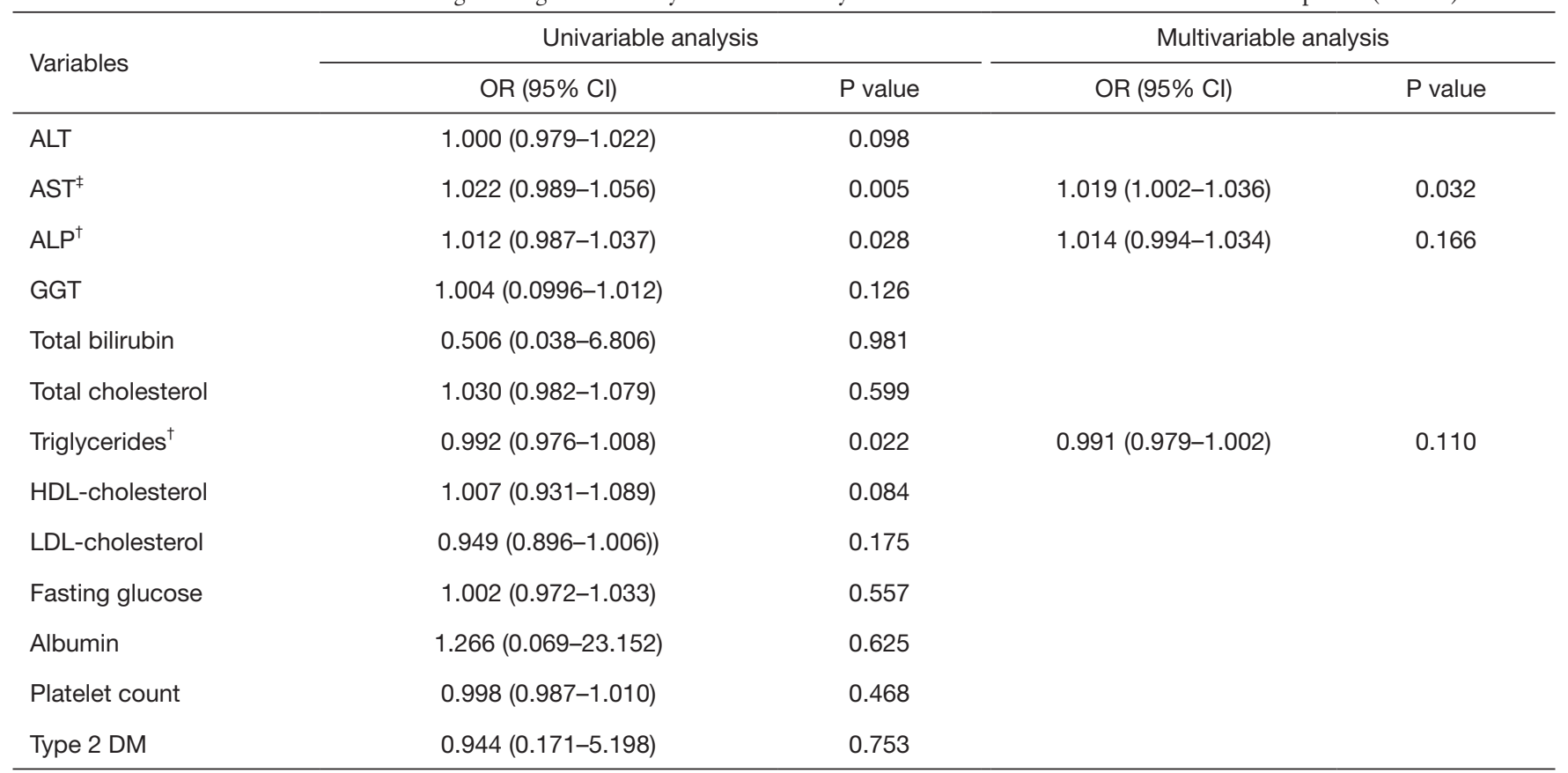

Data in brackets are 95\% confidence intervals. ${ }^{\dagger}$, Significant factors assessed by univariable logistic regression analysis; ${ }^{\ddagger}$, Significant factors assessed by both univariable and multivariable logistic regression analyses. OR, odds ratio; Cl, confidence interval; ALT, alanine aminotransferase; AST, aspartate aminotransferase; ALP, alkaline phosphatase; GGT, $\gamma$-glutamyltransferase; HDL, high density lipoprotein; LDL, low density lipoprotein; DM, diabetes mellitus.

Table 5 Diagnostic performance of ultrasonography (US) indices for the detection of non-alcoholic steatohepatitis (NASH) in non-alcoholic fatty liver disease (NAFLD) patients

\begin{tabular}{lcccc}
\hline Index & AUC & Accuracy (\%) & Sensitivity (\%) & Specificity (\%) \\
\hline Gray-scale US index & $0.794(0.670-0.888)$ & $78.3(65.8-87.9)$ & $68.0(46.5-85.1)$ & $85.7(69.7-95.2)$ \\
Fibroscan index & $0.687(0.554-0.800)$ & $71.67(58.6-82.6)$ & $48.0(27.8-68.7)$ & $88.6(73.3-96.8)$ \\
(Gray-scale US + AST) index & $0.806(0.683-0.897)$ & $75.0(62.1-85.3)$ & $68.0(46.5-85.1)$ & $80.0(63.1-91.6)$ \\
(Fibroscan + AST) index & $0.743(0.614-0.847)$ & $75.0(62.1-85.3)$ & $44.0(24.4-65.1)$ & $97.1(85.1-99.9)$ \\
(Gray-scale US + Fibroscan) index & $0.786(0.661-0.882)$ & $80.0(67.7-89.2)$ & $72.0(50.6-87.9)$ & $85.7(69.7-95.2)$ \\
(Gray-scale US + Fibroscan + AST) index & $0.798(0.674-0.890)$ & $80.0(67.7-89.2)$ & $56.0(34.9-75.6)$ & $97.1(85.1-99.9)$ \\
\hline
\end{tabular}

Data in brackets are 95\% confidence intervals. AUC, area under the curve; AST, aspartate aminotransferase.

NAFLD patients. Among them, the US index combing gray-scale US parameters and AST demonstrated the best diagnostic performance (AUC, 0.806). SWE and TE demonstrated high diagnostic performance for grading hepatic fibrosis (mean AUC, 0.777 and 0.830, respectively) and ballooning degeneration (mean AUC, 0.729 and 0.708, respectively) in NAFLD patients. The HRI also showed high diagnostic performance for grading hepatic steatosis (mean AUC, 0.871) and significantly higher diagnostic performance than CAP. Among laboratory data, AST was the only significant factor for predicting NASH (OR, 1.019; $\mathrm{P}=0.032$ ). AST showed high diagnostic performance for grading lobular inflammation (mean AUC, 0.712) and ballooning degeneration (mean AUC, 0.775). Although SWE, TE, HRI, and AST showed high diagnostic performance for staging fibrosis, steatosis, lobular inflammation, and ballooning degeneration, it is difficult to predict $\mathrm{NASH}$ with a single imaging modality, given 


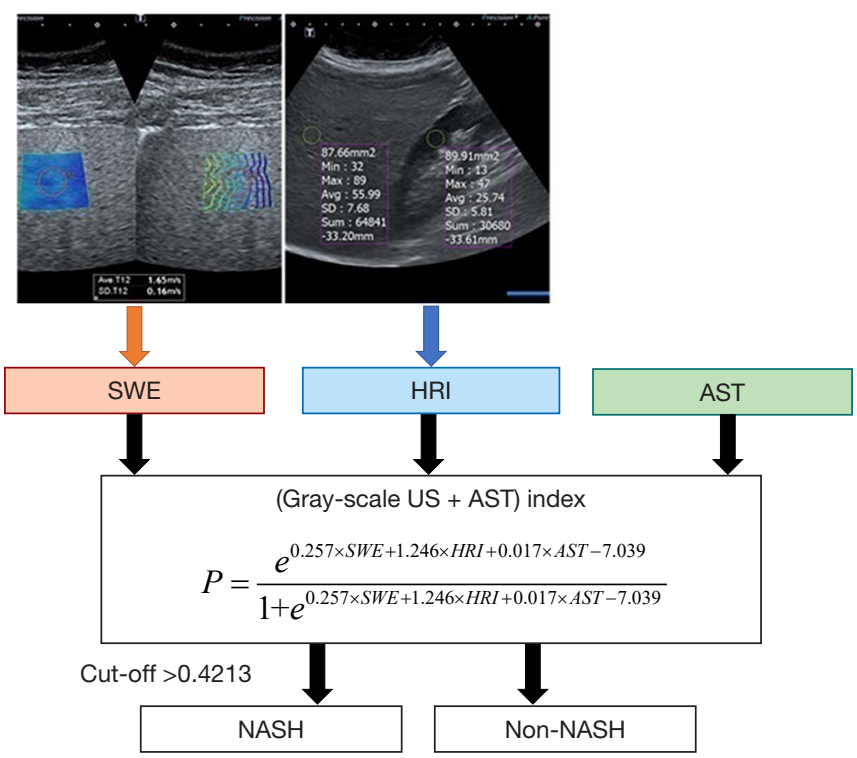

Figure 3 The index combining gray-scale ultrasonography (US) parameters and aspartate aminotransferase (AST). Based on the multivariable logistic regression model, the US index consisting of gray-scale US parameters (SWE and HRI) and AST was determined in the form of predicted probability $(\mathrm{P})$. The optimal cutoff value was calculated as $>0.4213$ which determines whether NASH is or not. SWE, shear-wave elastography; HRI, hepatorenal index; AST, aspartate aminotransferase; NASH, non-alcoholic steatohepatitis.

that it is composed of various histopathologic components (steatosis, ballooning degeneration, lobular inflammation, and fibrosis). Therefore, we devised US indices that combines US parameters and AST. SWE and the HRI can be measured on the gray-scale US, while TE and CAP can be measured on the Fibroscan. We developed various US indices combining gray-scale US parameters (SWE and HRI) and/or Fibroscan parameters (TE and CAP) with or without AST. The index consisting of gray-scale US parameters and AST showed the highest AUC than other indices. Our new US index combining SWE, HRI, and AST might be used to exclude the need for liver biopsy in NAFLD patients through further studies. Through this study, we carefully propose that additional Fibroscan may not be necessary to measure the liver stiffness and fat in NAFLD patients.

In the clinical practice, it is crucial to identify NASH in NAFLD patients; thus, there have been many attempts to develop non-invasive methods of predicting NASH. Several clinical prediction rules that combine demographic and laboratory data (e.g., FIB-4 index, BARD score, and NAFLD fibrosis score) have been developed for predicting fibrosis and cirrhosis in NAFLD patients (23). The major drawbacks, however, are the reduced accuracy in detecting early-stage fibrosis and the high proportion of patients with undetermined results (23). Elastography is emerging as one of the promising methods of staging hepatic fibrosis and diagnose NASH through the evaluation of the liver stiffness. MR elastography has the advantages of a larger sampling volume and less technical failure, but US elastography has been more widely used in clinical practice because of its convenience, shorter acquisition time, lower cost, and higher availability. US-based SWE can be divided into TE, 2D-SWE, and p-SWE $(24,25)$. TE is the first commercially available US-based elastography technique for the measurement of liver stiffness with one-dimensional probe (25). It has demonstrated excellent diagnostic performance for detecting advanced fibrosis and cirrhosis in NAFLD patients $(26,27)$. 2D-SWE and p-SWE are more recently developed US-based elastography and incorporated into conventional gray-scale US, allowing real-time measurement of liver stiffness (25). 2D-SWE also has shown good diagnostic performance for the diagnosis of various fibrosis stages in NAFLD patients $(28,29)$. There have been several studies that compare the diagnostic performance of TE and 2D-SWE for staging fibrosis in NAFLD patients $(28,30,31)$. In a previous meta-analysis including 156 NAFLD patients, 2D-SWE demonstrated significantly higher diagnostic performance than $\mathrm{TE}$ for the diagnosis of advanced fibrosis (12.8\% larger AUC, $\mathrm{P}=0.003)$, but not 

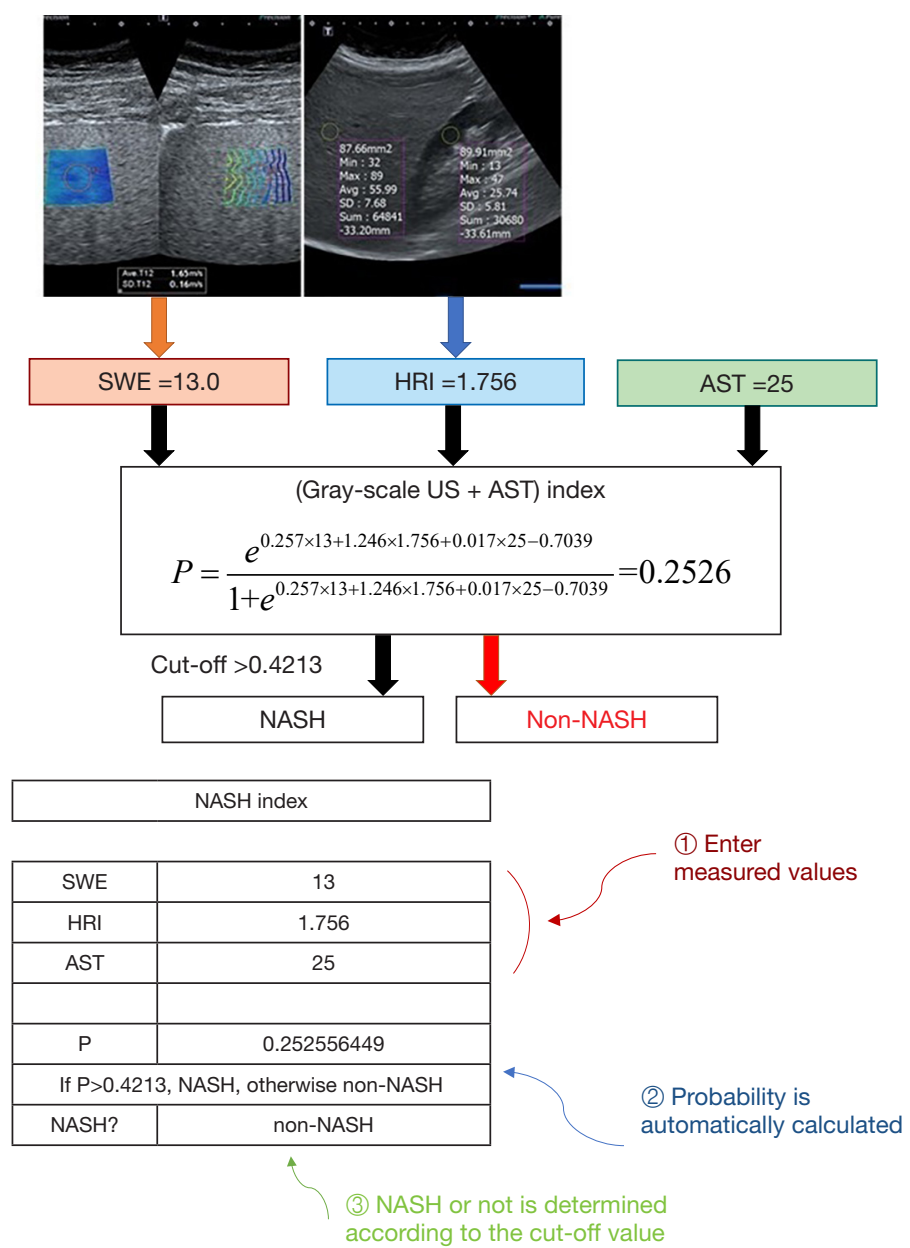

Figure 4 An example of using the ultrasonography (US) index to predict non-alcoholic steatohepatitis (NASH) in non-alcoholic fatty liver (NAFLD) patient. A 59-year-old woman with clinically suspected NASH who underwent percutaneous liver biopsy, gray-scale US, and Fibroscan. (A) Shear-wave elastography (SWE) and hepatorenal index (HRI) measured on the gray-scale US were $13.0 \mathrm{kPa}$ and 1.756 , respectively. Serum aspartate aminotransferase (AST) level was 25 IU/L. The predicted probability (P) calculated according to the US index was 0.2526 , which was less than the cutoff value of 4.213 , so this patient was predicted to be a non-NASH patient. The patient was actually classified as a non-NASH patient based on the histopathological analysis of the liver biopsy specimen according to the NAFLD activity score (NAS) (S1, L2, B0, and F0; NAS =3). (B) Because the probability equation was somewhat complex, we created a tool using Excel software that can easily predict NASH according to the pre-entered equation by entering variables.

for significant fibrosis $(6.4 \%$ larger AUC, $\mathrm{P}=0.162)$ and cirrhosis (6.7\% larger AUC, $\mathrm{P}=0.175)$ (28). In a previous prospective study involving 231 NAFLD patients, 2D-SWE and TE demonstrated similar diagnostic performance for detecting significant fibrosis (AUC, 0.85 vs. $0.83, \mathrm{P}=0.5$ ), advanced fibrosis (AUC, $0.87 v s$. $0.86, \mathrm{P}=0.5$ ), and cirrhosis (0.88 vs. $0.86, \mathrm{P}=0.5$ ) (30). In a recent prospective study involving 62 NAFLD patients, 2D-SWE and TE also showed comparable diagnostic performance in the detection of significant fibrosis (AUC, 0.80 vs. $0.77, \mathrm{P}=0.317$ ) and advanced fibrosis (AUC, 0.89 vs. 0.86, $\mathrm{P}=0.293$ ) (31). These results are similar to those of our study. In our study, there was no significant difference in the diagnostic performances of 2D-SWE and TE in the diagnosis of significant fibrosis (AUC, 0.747 vs. $0.869, \mathrm{P}=0.302$ ) and advanced fibrosis (AUC, 0.861 vs. 0.869, $\mathrm{P}=0.968$ ).

A variety of imaging modalities, including computed tomography (CT), US, MRI, and MRS have been used to evaluate NAFLD. Among them, US has several advantages over the others, including the absence of radiation exposure 
and availability. Therefore, US has been used as the primary imaging modality in evaluating liver diseases. The evaluation of liver fat using US mainly uses changes in the echogenicity of the liver parenchyma. As hepatic steatosis progresses through the intracellular accumulation of fat vacuoles, the echogenicity of the liver parenchyma increases and is higher ("brighter") than the renal cortex. US provides a fair accuracy for the diagnosis of the moderate to severe fatty liver, with a reported sensitivity of about $90 \%$ and specificity of about $95 \%$ (32). However, US shows relatively low accuracy in detecting mild fatty liver, and the reported sensitivity and specificity for all degrees of hepatic steatosis were $55.3 \%$ to $66.6 \%$ and $77.0 \%$ to $93.1 \%$, respectively (32). To overcome these limitations of US, there have been several attempts to establish methods for the quantitative assessment of hepatic fat using US. CAP is one of the recently developed US-based quantification methods of hepatic fat. It is obtained simultaneously with TE from the Fibroscan (Echosens, Paris, France). In a multicenter prospective study involving 404 patients, CAP identified patients with hepatic steatosis with an AUC of 0.87 for $\geq \mathrm{S} 1$, 0.77 for $\geq \mathrm{S} 2$, and 0.70 for $\geq \mathrm{S} 3$ (33). In a meta-analysis of CAP in suspected NAFLD patients, the mean AUCs for the diagnosis of $\geq S 1, \geq S 2$, and $\geq S 3$ steatosis were $0.96,0.82$, and 0.70 , respectively (34). The diagnostic performance is better for $\geq \mathrm{S} 1$ steatosis than for $\geq \mathrm{S} 2$ and $\geq \mathrm{S} 3$ steatosis. In our study, there was no patient with $\mathrm{S} 0$ steatosis from the histopathologic reports; therefore, we could not perform ROC curve analysis for the diagnosis of $\geq \mathrm{S} 1$ steatosis. The mean AUCs of CAP for $\geq \mathrm{S} 2$ and $\geq \mathrm{S} 3$ steatosis in our study were 0.616 and 0.549 , respectively, which were lower than the corresponding values from previous studies. The higher the degree of hepatic steatosis, the lower the diagnostic value of CAP. This may be due to the thick subcutaneous tissue. A previous study demonstrated that skin to capsular distance (SCD) $\geq 25 \mathrm{~mm}$ may cause overestimation of steatosis (35). In our study, 15 patients $(25 \%)$ had SCD $\geq 25 \mathrm{~mm}$. In addition, a relatively small number of patients in our study may have contributed to the lower diagnostic value of CAP. The HRI is another US-based quantification of hepatic steatosis. It is the ratio of the echo intensities of the liver parenchyma and the renal cortex. Previous studies have demonstrated an excellent correlation between the HRI and hepatic fat using MRS or liver biopsy as the reference standard, with reported AUC of about $99 \%(36,37)$. Previous studies using the HRI for sonographic screening of hepatic steatosis used an extrinsic software as well as an additional process of converting Digital Imaging and
Communications in Medicine (DICOM) images to Joint Photographic Experts Group images (36,38). However, a previous study demonstrated that the HRI could be quickly and accurately obtained from DICOM images on a PACS without additional software (21). Therefore, we used this direct method in our study to calculate the HRI.

This study has several limitations. First, this study was a retrospective study, therefore there might have been selection bias. We included only pathologically confirmed NAFLD/NASH patients. However, performing the liver biopsy in healthy people has an ethical problem. In our study, the prevalence of NASH in NAFLD patients were $41.7 \%$. In a previous meta-analysis reporting the epidemiology of NAFLD, the global prevalence of NASH in NALFD patients were reported to be $59.1 \%$ (95\% CI: 47.6-69.7\%) (1). In our study and studies of the metaanalysis, there may have been selection bias because NAFLD patients were selected as candidates for liver biopsy based on high suspicion for NASH. In addition, inter-reader reliability could not be assessed because SWE, $\mathrm{TE}$, and CAP were measured in real time in the past. Second, only a small number of patients in a single tertiary center using one US device were included. Our new devised US index was not validated using a validation group due to the small number of patients. To devise an US index which can be more widely used in a variety of clinical settings, the results of this study need to be validated using a validation group in a prospective study involving a larger number of patients and different US devices. In addition, serum TG is usually known to increase as the severity of NAFLD increases (39), but in our study, serum TG was higher in non-NASH patients than in NASH patients, which is also thought to be due to small number of patients. Similarly, some studies have shown that low density lipoprotein (LDL) cholesterol level was higher in NASH patients than in non-NASH patients $(40,41)$, but there was no significant difference in our study. Third, shear-wave dispersion slope recently demonstrated a high diagnostic performance for grading lobular inflammation in NAFLD patients (42). However, it is limited in use only in a newer version of US machine and not widely used in many institutions around the world. Instead, we used serum AST level which can be easily obtained from blood test anywhere in the world, as a parameter reflecting lobular inflammation and ballooning degeneration in this study. Similarly, attenuation coefficient parameters such as ultrasound-guided attenuation parameter (UGAP) have demonstrated high diagnostic accuracy for detecting and grading hepatic steatosis (43), 
but they are available only in a newer version of US machine. Therefore, we used HRI as a tool for evaluating hepatic steatosis that can be measured on all US machines. If shear-wave dispersion and UGAP are widely used in the future, it is expected to develop an index combining them for the detection of NASH in NAFLD patients.

\section{Conclusions}

Various US indices show good diagnostic performance in the detection of NASH in NAFLD patients. Among them, the US index that consists of gray-scale US parameters (SWE and HRI) and AST demonstrates the best diagnostic performance. SWE is available in most US machines, HRI can be easily calculated on all US machines, and AST can be easily obtained from blood tests. The US index combining gray-scale US parameters and AST might be used to exclude the need for liver biopsy in NAFLD patients.

\section{Acknowledgments}

This study was presented by the first author at RSNA 2018, Gastrointestinal (Liver Diffuse Disease, Steatosis) Session. Funding: None.

\section{Footnote}

Reporting Checklist: The authors have completed the STARD reporting checklist. Available at https://dx.doi. org/10.21037/qims-21-895

Conflicts of Interest: All authors have completed the ICMJE uniform disclosure form (available at https://dx.doi. org/10.21037/qims-21-895). CHL serves as an unpaid editorial board member of Quantitative Imaging in Medicine and Surgery. The other authors have no conflicts of interest to declare.

Ethical Statement: The authors are accountable for all aspects of the work in ensuring that questions related to the accuracy or integrity of any part of the work are appropriately investigated and resolved. The study was conducted in accordance with the Declaration of Helsinki (as revised in 2013). This study was reviewed and approved by the institutional review board (IRB) of the Korea University Guro Hospital (Approval No. KUGH16184). The requirement for informed consent was waived by the IRB considering the retrospective design of the study.
Open Access Statement: This is an Open Access article distributed in accordance with the Creative Commons Attribution-NonCommercial-NoDerivs 4.0 International License (CC BY-NC-ND 4.0), which permits the noncommercial replication and distribution of the article with the strict proviso that no changes or edits are made and the original work is properly cited (including links to both the formal publication through the relevant DOI and the license). See: https://creativecommons.org/licenses/by-nc-nd/4.0/.

\section{References}

1. Younossi ZM, Koenig AB, Abdelatif D, Fazel Y, Henry L, Wymer M. Global epidemiology of nonalcoholic fatty liver disease-Meta-analytic assessment of prevalence, incidence, and outcomes. Hepatology 2016;64:73-84.

2. Hsiao CC, Teng PH, Wu YJ, Shen YW, Mar GY, Wu FZ. Severe, but not mild to moderate, non-alcoholic fatty liver disease associated with increased risk of subclinical coronary atherosclerosis. BMC Cardiovasc Disord 2021;21:244.

3. Hsu CL, Wu FZ, Lin KH, Chen YH, Wu PC, Chen YH, Chen CS, Wang WH, Mar GY, Yu HC. Role of Fatty Liver Index and Metabolic Factors in the Prediction of Nonalcoholic Fatty Liver Disease in a Lean Population Receiving Health Checkup. Clin Transl Gastroenterol 2019;10:1-8.

4. Day CP. Natural history of NAFLD: remarkably benign in the absence of cirrhosis. Gastroenterology 2005;129:375-8.

5. Lee SS, Park SH. Radiologic evaluation of nonalcoholic fatty liver disease. World J Gastroenterol 2014;20:7392-402.

6. Ascha MS, Hanouneh IA, Lopez R, Tamimi TA, Feldstein AF, Zein NN. The incidence and risk factors of hepatocellular carcinoma in patients with nonalcoholic steatohepatitis. Hepatology 2010;51:1972-8.

7. Chalasani N, Younossi Z, Lavine JE, Diehl AM, Brunt EM, Cusi K, Charlton M, Sanyal AJ. The diagnosis and management of non-alcoholic fatty liver disease: practice Guideline by the American Association for the Study of Liver Diseases, American College of Gastroenterology, and the American Gastroenterological Association. Hepatology 2012;55:2005-23.

8. Romero-Gómez M, Zelber-Sagi S, Trenell M. Treatment of NAFLD with diet, physical activity and exercise. J Hepatol 2017;67:829-46.

9. Takahashi Y, Sugimoto K, Inui H, Fukusato T. Current pharmacological therapies for nonalcoholic fatty 
liver disease/nonalcoholic steatohepatitis. World J

Gastroenterol 2015;21:3777-85.

10. Chen J, Talwalkar JA, Yin M, Glaser KJ, Sanderson SO, Ehman RL. Early detection of nonalcoholic steatohepatitis in patients with nonalcoholic fatty liver disease by using MR elastography. Radiology 2011;259:749-56.

11. Gaidos JK, Hillner BE, Sanyal AJ. A decision analysis study of the value of a liver biopsy in nonalcoholic steatohepatitis. Liver Int 2008;28:650-8.

12. Cobbold JF, Patel D, Taylor-Robinson SD. Assessment of inflammation and fibrosis in non-alcoholic fatty liver disease by imaging-based techniques. J Gastroenterol Hepatol 2012;27:1281-92.

13. Yoneda M, Yoneda M, Mawatari H, Fujita K, Endo H, Iida $\mathrm{H}$, et al. Noninvasive assessment of liver fibrosis by measurement of stiffness in patients with nonalcoholic fatty liver disease (NAFLD). Dig Liver Dis 2008;40:371-8.

14. Yoon JH, Lee JM, Joo I, Lee ES, Sohn JY, Jang SK, Lee KB, Han JK, Choi BI. Hepatic fibrosis: prospective comparison of MR elastography and US shear-wave elastography for evaluation. Radiology 2014;273:772-82.

15. Sandrin L, Fourquet B, Hasquenoph JM, Yon S, Fournier C, Mal F, Christidis C, Ziol M, Poulet B, Kazemi F, Beaugrand M, Palau R. Transient elastography: a new noninvasive method for assessment of hepatic fibrosis. Ultrasound Med Biol 2003;29:1705-13.

16. Sigrist RMS, Liau J, Kaffas AE, Chammas MC, Willmann JK. Ultrasound Elastography: Review of Techniques and Clinical Applications. Theranostics 2017;7:1303-29.

17. Dasarathy S, Dasarathy J, Khiyami A, Joseph R, Lopez $\mathrm{R}$, McCullough AJ. Validity of real time ultrasound in the diagnosis of hepatic steatosis: a prospective study. J Hepatol 2009;51:1061-7.

18. van Werven JR, Marsman HA, Nederveen AJ, Smits NJ, ten Kate FJ, van Gulik TM, Stoker J. Assessment of hepatic steatosis in patients undergoing liver resection: comparison of US, CT, T1-weighted dual-echo MR imaging, and point-resolved $1 \mathrm{H}$ MR spectroscopy. Radiology 2010;256:159-68.

19. de Lédinghen V, Vergniol J, Foucher J, Merrouche W, le Bail B. Non-invasive diagnosis of liver steatosis using controlled attenuation parameter (CAP) and transient elastography. Liver Int 2012;32:911-8.

20. Kleiner DE, Brunt EM, Van Natta M, Behling C, Contos MJ, Cummings OW, Ferrell LD, Liu YC, Torbenson MS, Unalp-Arida A, Yeh M, McCullough AJ, Sanyal AJ; Nonalcoholic Steatohepatitis Clinical Research Network. Design and validation of a histological scoring system for nonalcoholic fatty liver disease. Hepatology 2005;41:1313-21.

21. Shiralkar K, Johnson S, Bluth EI, Marshall RH, Dornelles A, Gulotta PM. Improved method for calculating hepatic steatosis using the hepatorenal index. J Ultrasound Med 2015;34:1051-9.

22. Han K, Song K, Choi BW. How to Develop, Validate, and Compare Clinical Prediction Models Involving Radiological Parameters: Study Design and Statistical Methods. Korean J Radiol 2016;17:339-50.

23. Vilar-Gomez E, Chalasani N. Non-invasive assessment of non-alcoholic fatty liver disease: Clinical prediction rules and blood-based biomarkers. J Hepatol 2018;68:305-15.

24. Honda Y, Yoneda M, Imajo K, Nakajima A. Elastography Techniques for the Assessment of Liver Fibrosis in NonAlcoholic Fatty Liver Disease. Int J Mol Sci 2020;21:4039.

25. Chimoriya R, Piya MK, Simmons D, Ahlenstiel G, Ho V. The Use of Two-Dimensional Shear Wave Elastography in People with Obesity for the Assessment of Liver Fibrosis in Non-Alcoholic Fatty Liver Disease. J Clin Med 2020;10:95.

26. Jiang W, Huang S, Teng H, Wang P, Wu M, Zhou X, Ran H. Diagnostic accuracy of point shear wave elastography and transient elastography for staging hepatic fibrosis in patients with non-alcoholic fatty liver disease: a metaanalysis. BMJ Open 2018;8:e021787.

27. Kwok R, Tse YK, Wong GL, Ha Y, Lee AU, Ngu MC, Chan HL, Wong VW. Systematic review with metaanalysis: non-invasive assessment of non-alcoholic fatty liver disease--the role of transient elastography and plasma cytokeratin-18 fragments. Aliment Pharmacol Ther 2014;39:254-69.

28. Herrmann E, de Lédinghen V, Cassinotto C, Chu WC, Leung VY, Ferraioli G, et al. Assessment of biopsy-proven liver fibrosis by two-dimensional shear wave elastography: An individual patient data-based meta-analysis. Hepatology 2018;67:260-72.

29. Takeuchi H, Sugimoto K, Oshiro H, Iwatsuka K, Kono S, Yoshimasu Y, Kasai Y, Furuichi Y, Sakamaki K, Itoi T. Liver fibrosis: noninvasive assessment using supersonic shear imaging and FIB4 index in patients with nonalcoholic fatty liver disease. J Med Ultrason (2001) 2018;45:243-9.

30. Cassinotto C, Boursier J, de Lédinghen V, Lebigot J, Lapuyade B, Cales P, Hiriart JB, Michalak S, Bail BL, Cartier V, Mouries A, Oberti F, Fouchard-Hubert I, Vergniol J, Aubé C. Liver stiffness in nonalcoholic fatty liver disease: A comparison of supersonic shear imaging, 
FibroScan, and ARFI with liver biopsy. Hepatology 2016;63:1817-27.

31. Furlan A, Tublin ME, Yu L, Chopra KB, Lippello A, Behari J. Comparison of 2D Shear Wave Elastography, Transient Elastography, and MR Elastography for the Diagnosis of Fibrosis in Patients With Nonalcoholic Fatty Liver Disease. AJR Am J Roentgenol 2020;214:W20-6.

32. Lee DH. Imaging evaluation of non-alcoholic fatty liver disease: focused on quantification. Clin Mol Hepatol 2017;23:290-301.

33. Eddowes PJ, Sasso M, Allison M, Tsochatzis E, Anstee QM, Sheridan D, Guha IN, Cobbold JF, Deeks JJ, Paradis V, Bedossa P, Newsome PN. Accuracy of FibroScan Controlled Attenuation Parameter and Liver Stiffness Measurement in Assessing Steatosis and Fibrosis in Patients With Nonalcoholic Fatty Liver Disease. Gastroenterology 2019;156:1717-30.

34. Pu K, Wang Y, Bai S, Wei H, Zhou Y, Fan J, Qiao L. Diagnostic accuracy of controlled attenuation parameter (CAP) as a non-invasive test for steatosis in suspected nonalcoholic fatty liver disease: a systematic review and metaanalysis. BMC Gastroenterol 2019;19:51.

35. Shen F, Zheng RD, Shi JP, Mi YQ, Chen GF, Hu X, Liu YG, Wang XY, Pan Q, Chen GY, Chen JN, Xu L, Zhang RN, Xu LM, Fan JG. Impact of skin capsular distance on the performance of controlled attenuation parameter in patients with chronic liver disease. Liver Int 2015;35:2392-400.

36. Webb M, Yeshua H, Zelber-Sagi S, Santo E, Brazowski E, Halpern Z, Oren R. Diagnostic value of a computerized hepatorenal index for sonographic quantification of liver steatosis. AJR Am J Roentgenol 2009;192:909-14.

37. Mancini M, Prinster A, Annuzzi G, Liuzzi R, Giacco R, Medagli C, Cremone M, Clemente G, Maurea S, Riccardi G, Rivellese AA, Salvatore M. Sonographic hepaticrenal ratio as indicator of hepatic steatosis: comparison with (1)H magnetic resonance spectroscopy. Metabolism 2009;58:1724-30.

38. Marshall RH, Eissa M, Bluth EI, Gulotta PM, Davis NK. Hepatorenal index as an accurate, simple, and effective tool in screening for steatosis. AJR Am J Roentgenol 2012;199:997-1002.

39. Kashyap SR, Diab DL, Baker AR, Yerian L, Bajaj H, Gray-McGuire C, Schauer PR, Gupta M, Feldstein AE, Hazen SL, Stein CM. Triglyceride levels and not adipokine concentrations are closely related to severity of nonalcoholic fatty liver disease in an obesity surgery cohort. Obesity (Silver Spring) 2009;17:1696-701.

40. Imajo K, Hyogo H, Yoneda M, Honda Y, Kessoku T, Tomeno W, Ogawa Y, Taguri M, Mawatari H, Nozaki Y, Fujita K, Kirikoshi H, Saito S, Sumida Y, Ono M, Wada K, Nakajima A, Eguchi Y. LDL-migration index (LDL$\mathrm{MI})$, an indicator of small dense low-density lipoprotein (sdLDL), is higher in non-alcoholic steatohepatitis than in non-alcoholic fatty liver: a multicenter cross-sectional study. PLoS One 2014;9:e115403.

41. Sonmez A, Nikolic D, Dogru T, Ercin CN, Genc H, Cesur M, Tapan S, Karslioğlu Y, Montalto G, Banach M, Toth PP, Bagci S, Rizzo M. Low- and high-density lipoprotein subclasses in subjects with nonalcoholic fatty liver disease. J Clin Lipidol 2015;9:576-82.

42. Sugimoto K, Moriyasu F, Oshiro H, Takeuchi H, Abe M, Yoshimasu Y, Kasai Y, Sakamaki K, Hara T, Itoi T. The Role of Multiparametric US of the Liver for the Evaluation of Nonalcoholic Steatohepatitis. Radiology 2020;296:532-40.

43. Fujiwara Y, Kuroda H, Abe T, Ishida K, Oguri T, Noguchi S, Sugai T, Kamiyama N, Takikawa Y. The B-Mode Image-Guided Ultrasound Attenuation Parameter Accurately Detects Hepatic Steatosis in Chronic Liver Disease. Ultrasound Med Biol 2018;44:2223-32.
Cite this article as: Kim JW, Lee CH, Kim BH, Lee YS, Hwang SY, Park BN, Park YS. Ultrasonographic index for the diagnosis of non-alcoholic steatohepatitis in patients with non-alcoholic fatty liver disease. Quant Imaging Med Surg 2022;12(3):1815-1829. doi: 10.21037/qims-21-895 